\title{
Transit-time aspects of ENA production models for the inner heliosheath
}

\author{
M. Siewert ${ }^{1}$, H.-J. Fahr ${ }^{1}$, and D. J. McComas ${ }^{2,3}$ \\ 1 Argelander Institut für Astronomie der Universität Bonn, Abteilung f. Astrophysik und Extraterrestrische Forschung, \\ auf dem Hügel 71, 53121 Bonn, Germany \\ 2 Southwest Research Institute, San Antonio TX, USA \\ 3 Department of Physics and Astronomy, University of Texas, San Antonio TX, USA
}

Received 29 October 2013 / Accepted 17 February 2014

\section{ABSTRACT}

\begin{abstract}
It is still a major challenge to understand the data of energetic neutral atoms (ENAs) that arrive from the outer heliosphere. These data are provided by the IBEX mission. With the most recent data covering three years of dynamical evolution, it is now possible to study temporal correlations with the solar cycle. We present in this paper for the first time an extended study dedicated to the transit-time delay between the plasma that is ejected from the Sun towards the outer heliosphere and the ENA fluxes that reach the detector after the solar wind protons undergo charge exchange processes in their distant production regions in the inner heliosheath. We derive transit-time delays caused by both general and non-trivial model-dependent contributions for arbitrary points in the inner heliosheath. In addition, we also calculate the average transit-time delay along the line-of-sight in selected directions, allowing to estimate how strongly an extended emission region can reduce the resolution that is possible with transit-time studies. We are able to demonstrate that ENAs generated beyond a certain characteristic streamline distance are mostly removed by charge exchange, and thus do not contribute significantly to the keV-ENA fluxes observed by IBEX. For this reason, transit-time delays do provide a viable tool for studying the impact of the solar cycle on ENA fluxes.
\end{abstract}

Key words. shock waves - plasmas - solar wind - Sun: heliosphere

\section{Introduction}

The Interstellar Boundary EXplorer (IBEX) mission (McComas et al. 2009b) has provided some of the most interesting data on the global physics of the outer heliosphere to date (McComas et al. 2009a, 2012b). Detecting energetic neutral atoms (ENAs) generated in the outer heliosphere provides a new window into the otherwise inaccessible plasma physics at the borders of the solar system. Since the first release of the IBEX data (McComas et al. 2009a; Schwadron et al. 2009; Funsten et al. 2009; Fuselier et al. 2009), it became obvious that essentially all existing models of the heliospheric interface disagreed with the new observational data. Many new models, ideas and concepts related to the outer heliosphere have been put forward since (see e.g. McComas et al. 2012a, 2013, or the summary in McComas et al. $2012 b$ ), but still no new consensus description. One significant complication that limits a quantitative comparision of these new models is the position of the ENA production region. ENAs generated in the inner heliosheath are directly modulated by the solar wind, which dominates all individual components (e.g. thermal solar wind, pick-up ions) of the plasma flow in this region (see e.g. Zank et al. 2010; Livadiotis et al. 2011; Siewert et al. 2013). In contrast to this, identifying a possible modulation of more distant sources beyond the heliopause is less trivial because of the specific configurations and assumptions used by models devoted to these sources (see e.g. Heerikhuisen et al. 2010; Schwadron \& McComas 2013).

However, even after sorting different ENA production models according to their general heliospheric source region, a comparison of models that work on the same region is still a difficult task, therefore a new and different approach that allows a comparision of these models, independent of most of the microphysics, is highly desirable. In this study, we present one aspect that may serve as such a distinguishing property for ENA source models, namely the transit-time delay. This is based on the wellaccepted idea that any time-dependent modulation of the plasma sources that generate the observed IBEX ENA fluxes will be reflected in ENA observations. However, quantitative calculations on these correlations are nearly non-existent. Model calculations and data studies that place an emphasis on time-dependent aspects inevitably need to consider transit-time aspects as well (McComas et al. 2010, 2012b; Reisenfeld et al. 2012; Allegrini et al. 2012). However, all prior studies made use of strongly simplified models for dynamic properties of the solar cycle and the resulting dynamics of the source plasma regions, which diminishes the impact of quantitative studies of dynamic correlations between the solar cycle and ENA fluxes. For example, one typical approximation found in these studies is that the entire dynamics is characterized by one specific representative point in the emission region, ignoring curved plasma flows and an extended charge exchange region with a wide variety of plasma conditions. The earliest study of this type, which only examined the first year of IBEX data (see e.g. McComas et al. 2010), even restricted itself to one globally fixed production distance for all directions of the sky. Considering that this kind of calculations strongly depends on model assumptions, it is practically impossible to derive a simple description that covers all models found in the literature. In this study, we first summarize the equations and formulae required to calculate transit-time delays in sufficiently general situations, which serve as "building blocks" that can then be used to study explicit models. Following this, we use this formalism to study our own model for ENA production in 
the inner heliosheath (Fahr et al. 2011; Siewert et al. 2012, 2013) that is based on established analytic models for relevant properties of the inner heliosheath, such as the termination shock (TS) geometry, the plasma flow profile in the inner heliosheath (iHS), and the heliopause (HP). As we demonstrate, the finite line-ofsight extent of the ENA production region reduces the amount of information that can be gained from transit-time and solar wind correlation studies, but nevertheless, it delivers a selection criterion between competing models, especially those with transheliopause ENA production sites.

Following this, we introduce additional boundary conditions that are not directly related to the transit-time problem, but that naturally enter the modeling. We introduce PUI-removal effects caused by ongoing charge exchange of PUIs during the transport along the streamline; this effectively limits the distance from the detector from which ENA production contributes significantly to the integrated ENA flux seen by IBEX. We found that there exists a characteristic "cutoff" distance beyond which, for reasons discussed later in this study, sources of ENAs of high energies (from keV-protons) generated by charge exchange can be largely ignored.

\section{General aspects of transit-time calculations}

We begin by summarizing the aspects of the calculations that are the same for all models. First, one has to consider that ENA production involves two different species of particles, energetic ions and neutral $\mathrm{H}$-atoms, which differ in their behaviour when moving in a background plasma, i.e. frozen-in magnetic fields.

Therefore, the most straightforward contribution to the transit-time delay in the production of ENAs from solar wind protons is the flight time of the ENAs themselves. Thanks to the absence of an electric charge, ENAs at sufficient distance to gravitating bodies propagate in a simple, straight line, resulting in an ENA propagation time $\tau_{\text {ENA }}$ of

$\tau_{\mathrm{ENA}}=\frac{s^{\prime}}{v_{\mathrm{ENA}}}$.

Here $s^{\prime}$ is the distance traveled and $v_{\text {ENA }}$ is the velocity of the neutral atoms. This equation can be modified using standard astrophysical units (velocity to kinetic energy in $\mathrm{keV}$, meters to $\mathrm{AU})$, resulting in

$\tau_{\mathrm{ENA}}=1.08 \times 10^{-2}\left(\frac{E}{\mathrm{keV}}\right)^{-1 / 2}\left(\frac{s}{\mathrm{~cm}}\right) \mathrm{yr}$,

which means that, for example, a $1 \mathrm{keV}$ ENA requires 1.08 years (or $\simeq 394$ days) to travel a characteristic heliospheric distance of $100 \mathrm{AU}$. Considering that the energy range of IBEX-Hi covers an interval from 0.5 to $6 \mathrm{keV}$, this quantity can only be interpreted as an order-of-magnitude estimate.

Travel-times for ions are more complicated. Ions with energies of a few $\mathrm{keV}$ are bound to the global plasma flow, and therefore, they can only travel with the local solar wind bulk speed $v_{\mathrm{sw}}(\boldsymbol{x})$. In the inner heliosphere, which is dominated by the radial supersonic solar wind, one can adopt a fixed solar wind speed and obtain the simple equation

$\tau_{\mathrm{p}, \mathrm{sw}}=\frac{s}{v_{\mathrm{sw}}}$,

which is formally identical to Eq. (1). The velocity of the solar wind is, depending on the current phase of the solar cycle and the flow latitude, between $v_{\mathrm{sw}} \simeq 350-800 \mathrm{~km} \mathrm{~s}^{-1}$, which approximately corresponds to a mean thermal proton kinetic energy of
$1 \mathrm{keV}$, that is, we can adopt the same order-of-magnitude estimate as for the ENAs; however, the radial supersonic solar wind contribution to the accumulated transit-time does not depend on the ion energy. Strictly speaking, one also has to consider a deceleration of the solar wind due to mass-loading by pick-up ion generation and possibly other effects as well; observations clearly show that the solar wind does decelerate $10 \%$ from 1 AU to the termination shock (Le Roux \& Fichtner 1997; Fahr \& Ruciński 1999; Fahr 2007). Only 1 AU upstream of the termination shock, another deceleration occurs, reducing the solar wind speed by another $25 \%$ (Richardson et al. 2008). Including these corrections into our modeling requires integrating the transittime along the radial solar wind outflow using an apropriate transport equation (see e.g. Fahr \& Fichtner 2011) in the framework of the formalism we introduce for the inner heliosheath plasma flow in the next paragraph. However, fully quantitative calculations of these aspects in a realistic heliospheric model are not the main point of this study.

Another important aspect of the solar wind speed is its shorttime behavior, which may become important for the studies performed here in regions close to the polar regions, where the temporal resolution of IBEX is much shorter than six months (see e.g. Reisenfeld et al. 2012). As demonstrated for instance by Burlaga et al. (1996), the solar wind speed varies, which suggests that this behavior may limit the applicability of this study. However, we also recall that at solar distances of more than 5-10 AU, the solar wind is well described by an ideal supersonic fluid, which means that scattering and other energy or momentum exchange processes are negligible. Therefore, for the major part of the ion travel-time, it is possible to assume that individual "packets" of plasma that travels at constant velocity can be studied independent by each other, including two packets with slightly different velocities that intersect each other for some time. From these arguments it follows that for our first study of these aspects, it is sufficient to study the behavior of plasma packets that travel at a constant average speed.

It is observationally evident that the solar speed at some distance varies in time in the sense that it fluctuates around an average value $U$. One can investigate these fluctuations oin more detail and study variation of the fluctuation amplitude $\Delta U$ with distance $r$. In-situ observations of Voyager- 2 in regions between 10 and $40 \mathrm{AU}$ (Richardson et al. 1995) show that the average solar wind speed does not change with distance compared with solar wind bulk velocity measurements carried out with IMP-8 simultaneously at $1 \mathrm{AU}$. However the amplitude of the speed fluctuations strongly decreases with solar distance (see Fig. 6 of Richardson et al. 1995). This indicates from observations that the average speed of a wind with a fluctuating speed practically does not change, while the amplitude of the speed fluctuations clearly decreases with solar distance. The theoretical approach derived below thus makes use of these observations by adopting that the average solar wind speed $U=\langle U+\Delta U\rangle_{t}$ is constant with distance, except for a small overall deceleration caused by PUI mass and momentum loading; for the commonly accepted value of the local interstellar cold hydrogen density $n_{\mathrm{H}}=0.1 \mathrm{~cm}^{-3}$, this deceleration is about $40 \mathrm{~km} \mathrm{~s}^{-1}$ over $100 \mathrm{AU}$ (Fahr 2007), which is less than $10 \%$ of the slow solar wind speed, which means that this effect can be safely averaged out as well.

The most important nontrivial contribution to the transittime is the energetic ion propagation in the inner heliosheath downstream of the TS. Because of the interaction with the local interstellar medium, which gives rise to a tangential discontinuity, that is, the heliopause, the resulting plasma flow lines there are curved, and the transit-time cannot be calculated in a 


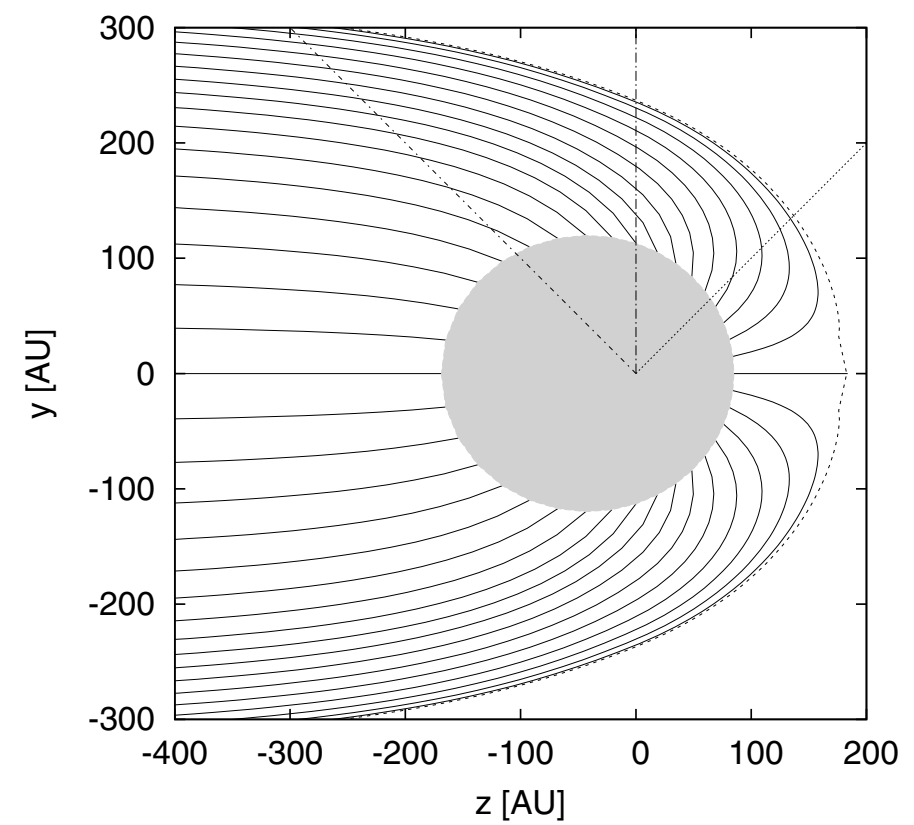

Fig. 1. Plasma flow lines in the inner heliosheath according to the Parker model modified by Fahr \& Fichtner (1991). Upwind direction points to the right, lines extending from the heliocenter denote lines of sight studied in Sect. 2.2.

straightforward manner. If one had an explicit parameterization of a streamline that crosses a specific point in the inner HS, calculating the transit-time contribution and other resulting properties would be trivial; unfortunately, such a parameterisation does not exist. The earliest model found in the literature to calculate inner HS streamlines, was reported by Parker (1965), who described the streamlines using a flow potential, which results in a velocity field on the downstream side that is derived from the gradient of a flow potential. An explicit calculation of streamlines then requires solving a differential equation numerically, i.e. beginning at the heliospheric TS, integrating the streamline according to the streamline potential while additionally keeping track of the time. While in principle capable of calculating streamlines in the inner HS, numerical models have not presented similar calculations in the literature, and therefore, we are unable to calculate contributions to the transit-time with the help of such models. To repeat the present study for numerical models requires representing of the flow line profile in parameterized form.

In the following study, we adopt the model by Fahr \& Fichtner (1991), which is a second-order correction to the Parker flow model; a quantitative representation of streamlines in the ecliptic plane is presented in Fig. 1.

\subsection{Isochronal maps of full transit-time delays}

First, we derived maps that provide the transit-times required by a proton traveling with the bulk solar wind from the Sun to the TS, to travel outwards in the inner heliosheath to some specific point where it undergoes charge exchange and returns to the observer as an ENA. Calulating these three individual contributions, the transit-time is (see also Fig. 2)

$$
\begin{aligned}
\tau_{\mathrm{tt}} & =\tau_{\mathrm{p}, \mathrm{sw}}+\tau_{\mathrm{p}, \mathrm{iHS}}+\tau_{\mathrm{ENA}} \\
& =\frac{r_{\mathrm{TS}}}{v_{\mathrm{sw}}}+\tau_{\mathrm{p}, \mathrm{iHS}}+\frac{s^{\prime}}{v_{\mathrm{ENA}}}
\end{aligned}
$$

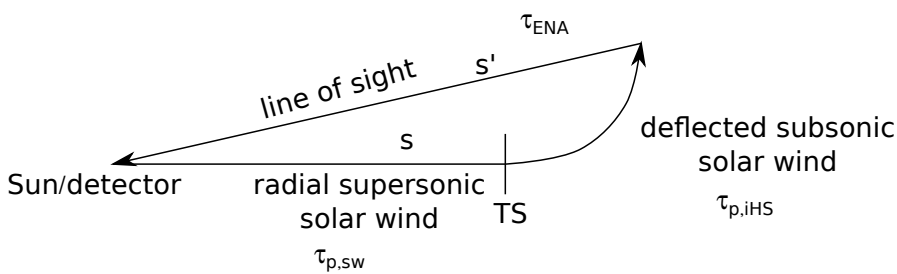

Fig. 2. Schematic representation of the path taken by the individual ions/ENAs from the Sun to the detector. Note that the ENA will usually return from a different direction than the one the parent ion took when moving outwards.

where $r_{\mathrm{TS}}$ is the radial distance from the Sun to the TS, $s^{\prime}$ is the distance from the proton-to-ENA conversion point to the detector, and $\tau_{\mathrm{p}, \mathrm{iHS}}$ is the (model-dependent) time required by the energetic ion to be transported from the downstream side of the TS to the conversion point, along the streamline connecting these two points. A schematic representation of these individual time contributions is presented in Fig. 2.

Any of these time scales for a reference point following a flow line with a velocity $\boldsymbol{U}(s)$ can be calculated by evaluating the integral

$\tau_{12}=\int_{1}^{2} \frac{\mathrm{d} s}{U(s)}$,

where $\mathrm{d} s$ is a parameterization of the path traveled by the reference point. For the first and third term in Eq. (4), the path traveled is a straight line and the travel speed $v$ is constant. With the exception of solar-cycle-induced velocity variations and a slight slowing-down of the solar wind caused by mass loading (both of which we do not consider in this study), the supersonic solar wind speed that enters the calculation of $\tau_{\mathrm{p}, \mathrm{sw}}$ is basically constant in the inner heliosphere along the radial outflow, which allows us to adopt an apropriate value for $v_{\mathrm{sw}}$. The third term, $\tau_{\text {ENA }}$, is also characterized by a constant ENA flow speed $v_{\text {ENA }}$, because electrically neutral atoms are not accelerated, decelerated, or deflected by electromagnetic forces. Therefore, the main contribution to this effect comes from $\tau_{\mathrm{p}, \mathrm{iHS}}$, for which Eq. (5) must be evaluated explicitly.

One of the first attempts to calculate these time scales in the context of ENA production processes and the IBEX data was presented by Reisenfeld et al. (2012), who assumed that energetic protons are basically continuing their purely radial outflow, with a downstream plasma flow speed equivalent to the local velocity of sound on the downstream side of the shock, without any modification by a deflection of streamlines. This approach is a major simplification of the actual plasma flow profile and only provides a good approximation for two specific directions in the nose and tail directions, but fails for the global heliosphere. A more realistic travel-time for a subsonic plasma that follows the stream lines into the upwind direction $\left(\tau_{\mathrm{p}, \mathrm{iHS}}\right)$ has been calculated by Chalov et al. (2007), who found that plasma entering the inner HS in the upwind direction, that is, along the stagnation line, can take a very long time, up to about 100 years, to reach an off-axis distance of $100 \mathrm{AU}$ or the flanks of the heliosheath.

In this study we present transit-times in the form of isochronal lines, that is, lines representing ENA production regions with the same transit-time delay. This allows us to present transit-times in a unified way, demonstrating from a global change in the solar wind that different regions on the sky posess significantly different time delays. Figure 3 shows this behavior by presenting isochrochronal lines based on different model parameters. Figure $3 \mathrm{a}$ presents results for $1 \mathrm{keV}$ ENAs and an 


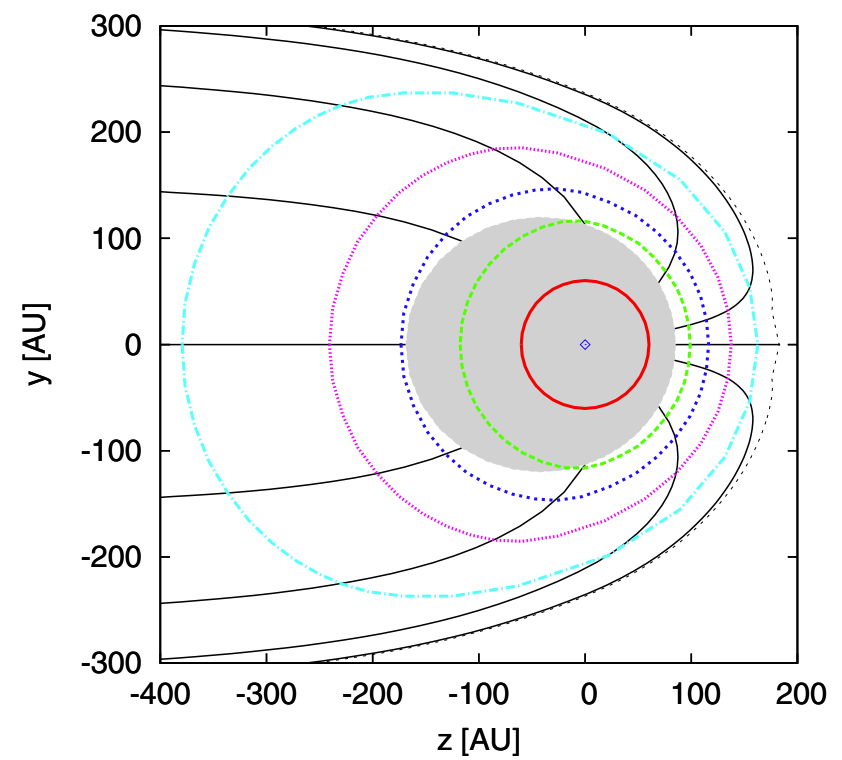

(a) $1 \mathrm{keV}$, fast SW

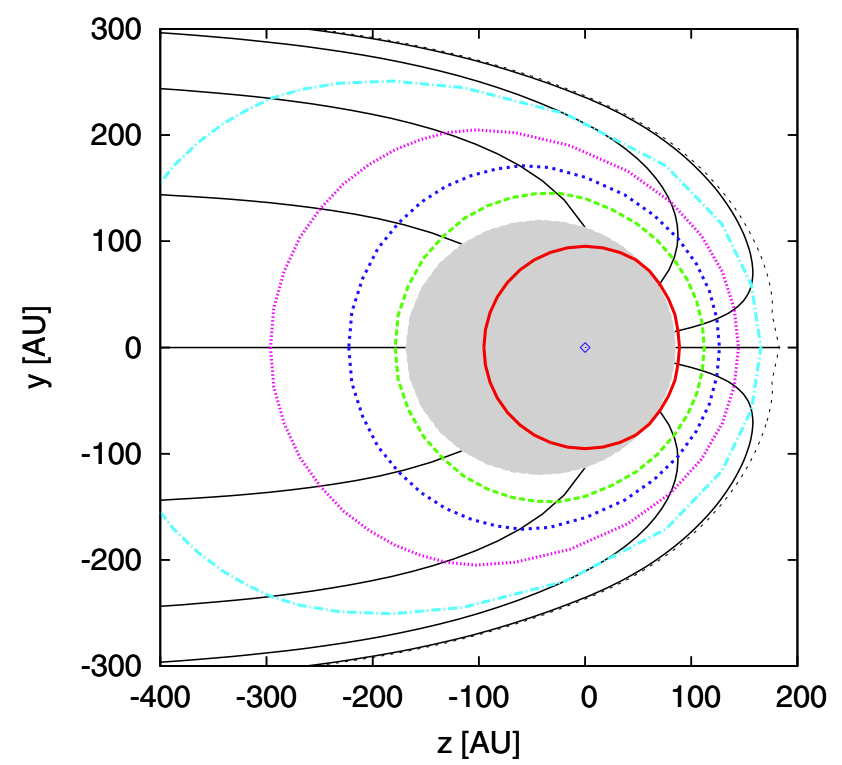

(c) $6 \mathrm{keV}$, fast SW

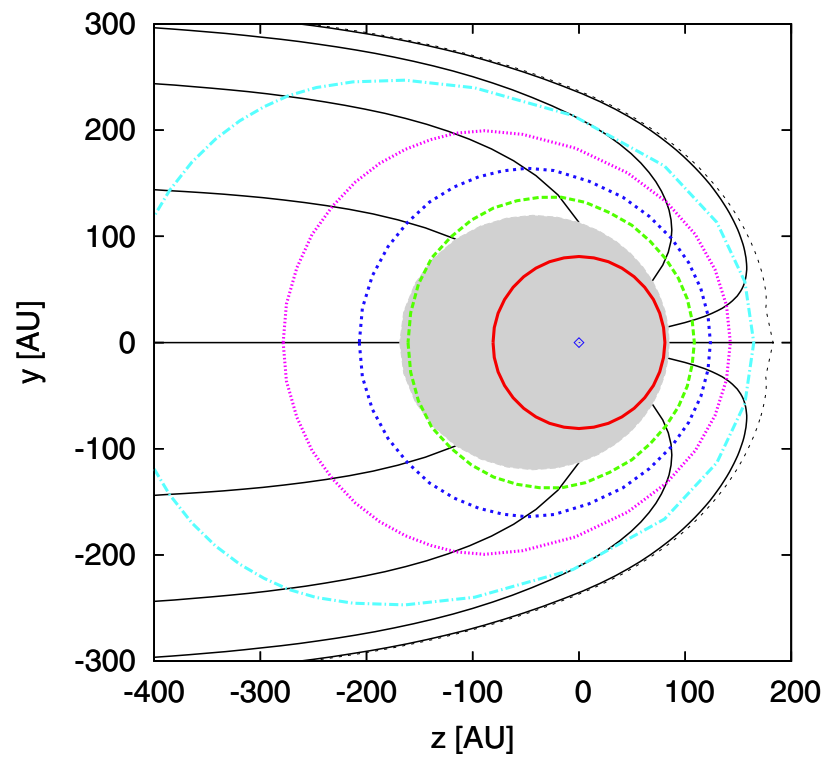

(b) $3 \mathrm{keV}$, fast $\mathrm{SW}$

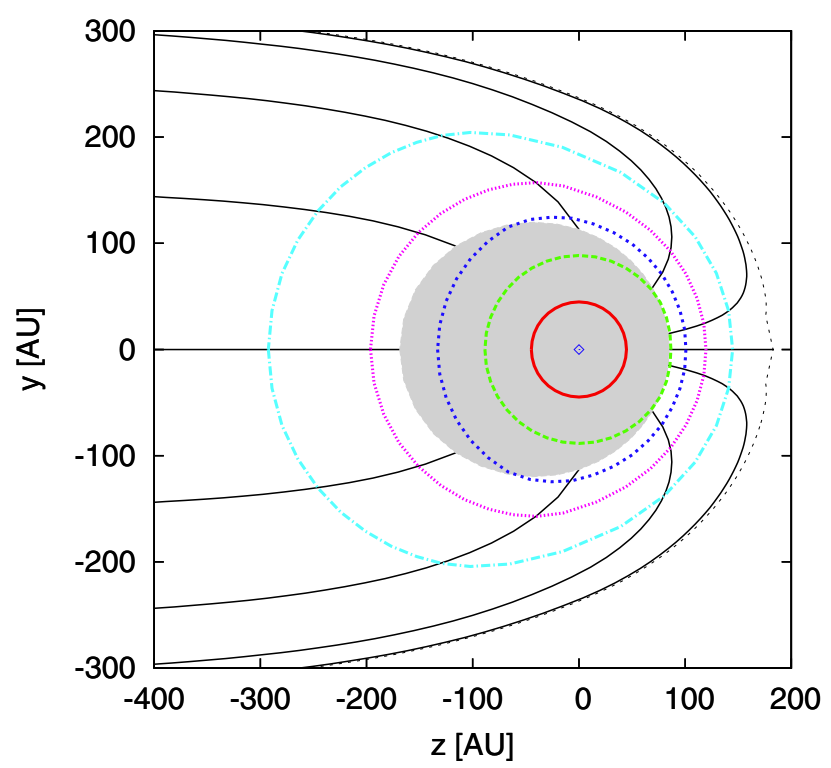

(d) $1 \mathrm{keV}$, slow $\mathrm{SW}$

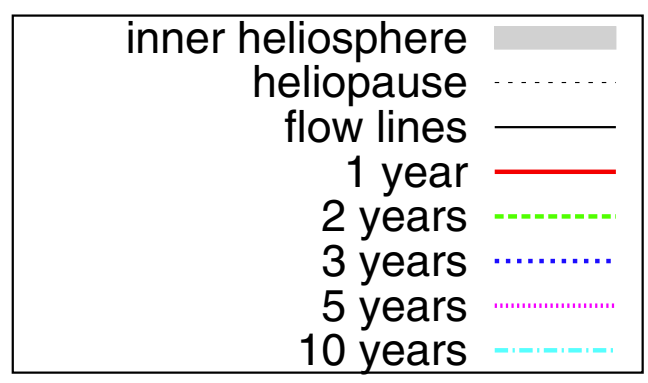

Fig. 3. Isochronal lines for various energies and solar wind speeds: the transit-time required by a SW proton observed close to the Sun after being emitted from the corona into the solar wind, crossing a certain distance in the inner HS before undergoing charge exchange, and returning as ENAs to the detector. Displayed lines denote the regions where the charge exchange happens, black lines are flow lines.

upstream solar wind speed of $v_{\mathrm{sw}} \simeq 760 \mathrm{~km} \mathrm{~s}^{-1}$, which is the highest velocity observed at high latitudes around a solar minimum (see e.g. McComas et al. 2000) heliospheric solar wind. In Fig. $3 \mathrm{~b}$ and c, we increase the ENA energy to 3 and $6 \mathrm{keV}$, which results in shorter transit-times because of a reduction of $\tau_{\text {ENA }}$ (see Eq. (1)); obviously, faster ENAs result in shorter traveltimes. In Fig. 3d, we again selected an ENA energy at $1 \mathrm{keV}$, while reducing the solar wind speed to typical slow solar wind 
values of $v_{\mathrm{sw}} \simeq 400 \mathrm{~km} \mathrm{~s}^{-1}$. This reduces the solar wind speed by a factor of 1.9 , resulting in an approximate doubling of $\tau_{\mathrm{p}, \mathrm{sw}}$ and an accordingly increased total transit-time delay.

These figures show a result obtained by Chalov et al. (2007), who found that the travel-time for the plasma streamlines in the inner heliosheath can grow very long when they approach the heliopause. For streamlines that are closest to the heliopause, travel speeds were found to be on the order of $1 \mathrm{AU} / \mathrm{year}$, which means that ENAs generated in these regions are associated with very long delay times.

By comparing the individual images, we are able to derive some rough estimates for transit-time delays using different plasma flow models (see e.g. Zank et al. 2010). Most models include the basic deflection of plasma towards the heliotail. In the upwind direction and for a fast solar wind, ENAs will begin to reflect a solar event after a transit-time of approximately between 1 and 2 years (for $6 \mathrm{keV}$ and $1 \mathrm{keV}$ ENAs, respectively, from the closest point in the inner heliosheath). In contrast, ENAs from the downwind direction will require at least one additional year to show this behavior because of the increased heliocentric distances of their individual charge-exchange regions; for the most extreme situation, that is, comparing the shortest and the longest TS distances, the shortest reaction time differs by nearly two years. On the other hand, if one keeps the ENA reference energy and instead modifies the solar wind speed, one easily sees that reducing the solar wind speed by a factor of 2 also adds about one year to the reaction time.

Taken together, these results show that the inner heliosheath flow line model by Fahr \& Fichtner (1991) results in transit-time spreads over a few years, depending on the heliospheric direction and the solar wind properties that dominate this time period. Because the IBEX resampling time has a temporal map resolution of 0.5 year (shorter at the poles), the resulting differences in the transit-time delay in different directions to a global change in the solar wind should be observable. At the time of writing, three years of ENA data have been fully evaluated and made available to the general public, covering the observation time from 2009 to 2012, and reflecting the solar cycle from the time between 2005 and 2009. According to McComas et al. (2012b), the global observational data agrees well with ENAs generated in the global decline during the past unusually deep and long solar minimum, and an (as-of-yet unquantified) rise of the ENA fluxes is expected at some point in the near future.

\subsection{Transit-time spreads along a line-of-sight}

After demonstrating the greatly varying transit-time delays for different regions of the heliosphere, we now study the impact of transit-time calculations along an individual line-of-sight. Obviously, following a line-of-sight outwards from the detector crosses many different streamlines, thus sampling different transit-time delays. This effect reduces the temporal resolution that can be obtained from line-of-sight integrated measurements.

To quantify this effect, we now present the transit-time delay along a line-of-sight, including the region between the TS and the HP. From Fig. 4 one immediately sees that when integrating over ENAs generated along a line-of-sight, the observed ENAs cover vastly different periods of the solar cycle, which complicates correlating dynamics that appear in ENA observations with the dynamics of the solar wind. On the other hand, these calculations also prove that the transit-times that overlap along a lineof-sight are a function of the direction into which the detector is oriented. In Sect. 2.3, we present two mechanisms that allow one to cut off the line-of-sight integral after a finite distance, which

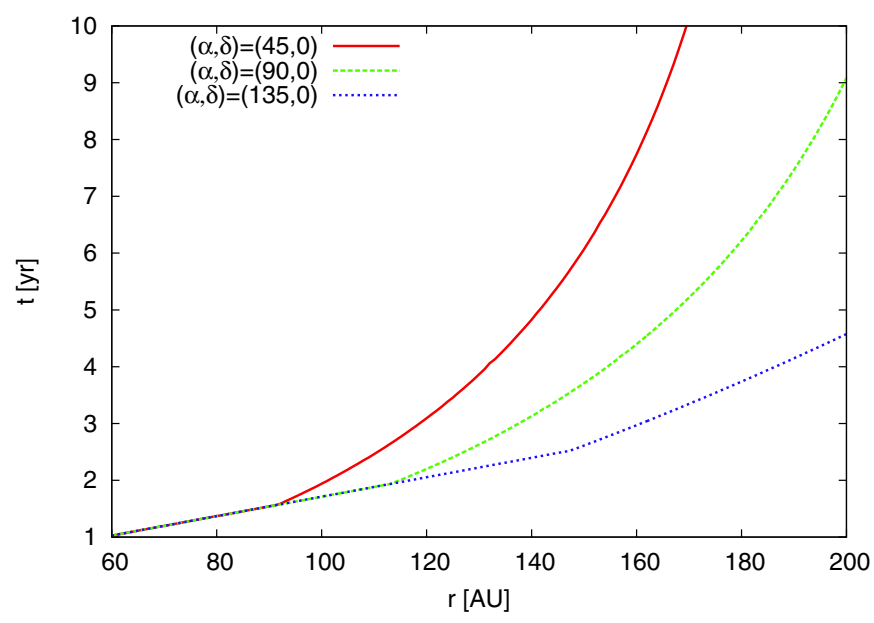

Fig. 4. Variable transit-time delay along a line-of-sight as a function of heliocentric distance $r$ from the Sun, caused by the line-of-sight crossing of many individual plasma streamlines with individual, different transit-times. As a baseline, we also display the linear inner heliosheath contribution, where no sufficient source population of energetic ions is found. The ENA energy is taken as $1 \mathrm{keV}$.

results in an upper limit for the transit-times that contribute signifcantly to the total ENA fluxes.

Before introducing this upper limit, another (technical) point must be taken into account. The IBEX detector uses broad energy channels that cover an extended energy window to generate intensities that are centered on a central energy value. Therefore, ENAs arriving at the lowest end of the energy band reflect different transit-time delays from ENAs at the upper end of the band. In addition, the source ENA distribution function provides different ion number densities, and thus in general (at least for soft distribution functions that describe continuously decreasing ion intensities for higher energies), more ENAs will be detected at the lower end of the energy band than at the higher end.

We studied this effect by plotting transit-time delays for the upper and lower end of the energy band for two selected energy bands of the IBEX detector. First, we used the 1-keV energy band, that is, the region where the statistics are the best and the ribbon feature is the clearest, located between 0.72 and $1.43 \mathrm{keV}$ (usually presented as the $1.08 \mathrm{keV}$ energy band). For the second energy band, we chose the $4.09 \mathrm{keV}$ energy band, that covers the range between 2.76 and $6 \mathrm{keV}$, which is a region where a reasonable statistics is still possible, and which does not overlap with the $1.08 \mathrm{keV}$ band. The resulting transit-times are presented in Fig. 5 for the upwind $\left((\alpha, \delta)=\left(45^{\circ}, 0^{\circ}\right)\right)$ line-of-sight studied earlier. As one can easily see from this figure, the uncertainty in the transit-times caused by a broad energy band in the detector is smaller than the uncertainty expected from the "different flow lines" effect.

\subsection{Removing ENA candidates along a flow line}

Following these straightforward considerations that increase the uncertainties in interpreting flux data in global dynamical studies, we now introduce an additional aspect of the transit-time problem that helps to reduce the time-convolution problem. In this section, we study an aspect that is tied to the energetic ion tail that serves as the primary source of ENAs. This aspect cannot be studied by conventional fluid descriptions such as MHD, which is commonly adopted by numerical modelers (see e.g. Heerikhuisen et al. 2007). 


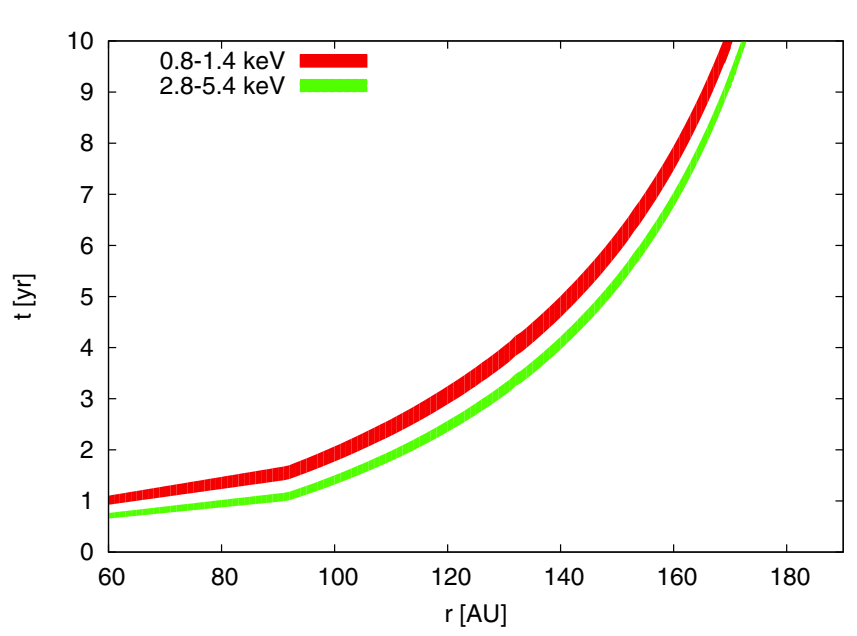

Fig. 5. Variable transit-time delay along a line-of-sight as a function of heliocentric distance $r$ from the Sun for two energy bands provided by IBEX-Hi.

It is a commonly known fact that MHD cannot describe in sufficient detail the behavior of a specific spectral population at a specific energy. For the energetic ion tails that we are interested in for the production of keV-ENAs, a more detailed explanation of this drawback has recently been given by Scudder \& Karimabadi (2013). Classical MHD is obtained by starting from a kinetic description and the subsequent introduction of so-called velocity moments, that is, a different, less detailed and informative representation of the distribution function. The moment equations that make up this description need a closure at a oneorder higher moment level; the highest order velocity moments most commonly used in the literature are pressures. Obviously, a more detailed, less approximate description of the plasma would require introducing higher order moments and appropriate additional terms in the equations. Energetic ions are represented by fourth-order velocity moments, that is, two full additional orders beyond standard MHD, and for this reason, it immediately follows that the IBEX modeling group (see e.g. Ratkiewicz et al. 1998; Heerikhuisen et al. 2007) did not include this detail into their MHD mode, which instead uses a standard, second-order MHD description, supported by a kinetic description that does not include the effects which we discuss now.

Owing to these complications, we calculated the following results using kinetic concepts instead of hydrodynamic ones, and we also did not not study all possible implications of the following calculations in depth. Schwadron et al. (2011) used similar arguments to perform a ribbon-separation study from the global ENA map, using hydrodynamic pressures and focusing on the heliotail, while we now perform a study that allows understanding a broader generality of heliospheric directions and spectral properties of the resulting ENAs. To understand the following arguments, one first has to consider the following simple concepts: ENAs produced in the inner heliosheath are generated by charge exchange processes with cold interstellar hydrogen,

$p^{*}+\mathrm{H} \rightarrow \mathrm{H}^{*}+p$,

where the asterisk denotes an energetic particle. This means that energetic ions are replaced by cold ions, which evidently reduces the spectral intensity of energetic ions in the specific plasma volume element, thus "cooling" the energetic tail of the downstream plasma flow, a concept that was described by Schwadron et al. (2011). Therefore, following any volume element advected with the wind along a flow line results in a systematic removal of energetic source ions from this element, until reaching a flow line distance where, due to the removal of energetic source ions, practically no keV-energetic ENAs are produced.

A detailed knowledge about the local ion distribution function at places downstream from the termination shock would in principle require solving a phase-space transport equation (see e.g. Fahr \& Fichtner 2011), which, however, for our purposes may not be needed in full generality due to the following arguments: first, we expect the heliosheath plasma to be characterized by fairly low effective Mach numbers $(M \ll 0.1)$, allowing the assumption that the heliosheath plasma flow is practically incompressible, and therefore, no adiabatic cooling occurs. Even though the Voyager observations of the TS suggest that the downstream plasma is still supersonic, this may be misleading because the Voyagers are unable to observe all plasma components of the solar wind. For example, several recent multicomponent studies of the solar wind (Wu et al. 2009; Zank et al. 2010) suggested that the downstream plasma is composed of thermal protons, nonthermal PUIs and also (Fahr \& Siewert 2013) energetic electrons, and that excludsing some of these components from the model may result in a strong overestimate of downstream Mach numbers. In fact, with hot electrons taken into account, effective downstream Mach numbers of $\bar{M}_{\mathrm{s} 2} \lesssim 10^{-1}$ are obtained, which allows us to assume incompressibility of the plasma. In addition, energy diffusion via wave-particle interaction requires isotropic nonlinear MHD turbulence, but in the region downstream of the shock one may only find unidirectional turbulence, and therefore, one can assume that energy diffusion is not important. Therefore, the transport equation practically describes a PUI distribution carried along a streamline that only changes due to differential charge exchange processes, which operate as pure loss-processes at high energies, and as pure gainprocesses at lower energies. For these reasons, it can therefore be assumed for ions at $\mathrm{keV}$ energies that the advected energetic ions only suffer charge-exchange-induced loss processes, while no significant sources for these energetic ions are active; the strongest source term consists of energetic ion regeneration following a charge exchange between an ENA and a solar wind ion, which would result in a source term proportional to $\sigma_{\mathrm{ex}}^{2}$, that is, negligible when compared with a sink term proportional to $\sigma_{\text {ex }}$.

As a result, when integrating ENAs produced along a line-ofsight, one has to consider that more distant regions have already lost their energetic ions at earlier locations. For this reason, it is possible to ignore ENA production after a certain line-of-sight distance. This effect must be quantified and taken into account in any calculation of ENA fluxes, which, to the best of our knowledge, has not been performed explicitly before.

To quantify this effect, we begin by writing down the extinction of energetic ions with an energy $E$ along a streamline,

$\frac{\partial f(E)}{\partial t}=-\sigma_{\mathrm{ex}}\left(v_{\mathrm{rel}}\right) n_{\mathrm{H}} v_{\text {rel }} f(E)$

where $v_{\text {rel }}$ is the relative speed difference between the energetic ions and the interstellar hydrogen atoms with density $n_{\mathrm{H}} . \sigma_{\mathrm{ex}}$ is the charge exchange cross section. This differential equation is solved by

$f(E, s)=f_{0}(E) \exp \left(-n_{\mathrm{H}} \int_{0}^{t} \sigma_{\text {ex }}\left(v_{\text {rel }}(\boldsymbol{u})\right) v_{\text {rel }} \mathrm{d} t^{\prime}\right)$,

where $f_{0}(E)$ is the distribution function on the downstream side of the TS, and $t$ the travel-time of the plasma along plasma flow line. $v_{\text {rel }}$ is the relative speed between the energetic ions and the cold interstellar hydrogen, and $\boldsymbol{U}(t)$ is the speed of the inner HS plasma flow while following the streamline. 
M. Siewert et al.: Transit-time aspects of ENA models

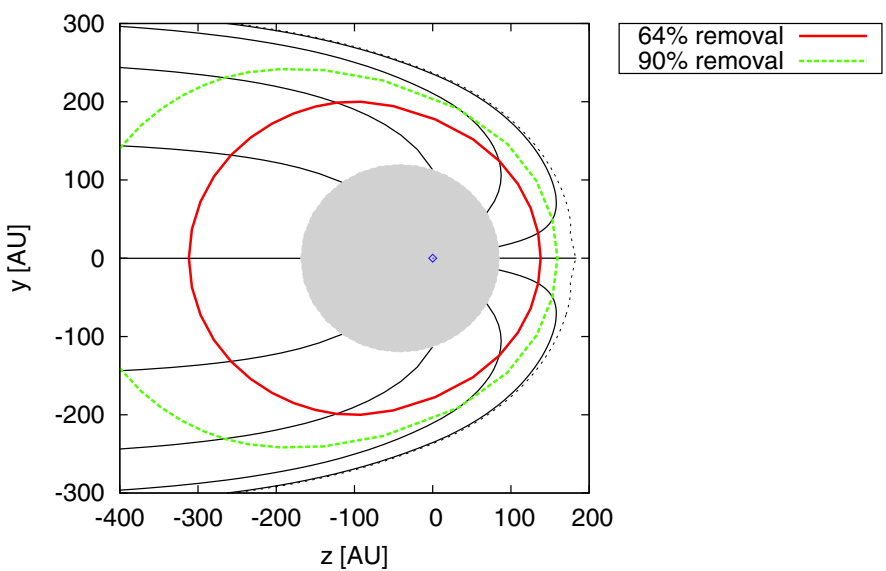

Fig. 6. Energetic proton extinction lines, denoting regions where a certain amount of $1 \mathrm{keV}$ ions has been removed by charge exchange.

The relative speed required to derive the correct crosssection must be derived from a vector-relation,

$\boldsymbol{v}_{\mathrm{rel}}=\boldsymbol{v}_{\mathrm{p}}+\boldsymbol{v}_{\mathrm{H}}$,

where the subscripts denote the proton and hydrogen velocities. The absolute value of this velocity is then given by

$v_{\text {rel }}=\sqrt{v_{\mathrm{p}}^{2}+v_{\mathrm{H}}^{2}+2 v_{\mathrm{p}} v_{\mathrm{H}} \cos \angle\left(\boldsymbol{v}_{\mathrm{p}}, \boldsymbol{v}_{\mathrm{H}}\right)}$.

The most recent value for the typical speed of the LISM hydrogen is about $23 \mathrm{~km} \mathrm{~s}^{-1}$ (McComas et al. 2012a), while the downstream ion speed is on the order of $100-200 \mathrm{~km} \mathrm{~s}^{-1}$, meaning that we can ignore the hydrogen speed for now and obtain the simplified expression

$v_{\text {rel }} \simeq\left|\boldsymbol{v}_{\mathrm{p}}\right|$.

Since we are only studying the general extinction effect, we do not investigate in detail of which individual contributions the proton velocity is composed or by which spectral properties it is modulated. Instead, we just calculate the survival factor due do ion removal $\epsilon(\boldsymbol{v}, \boldsymbol{x})$ which we define by

$\epsilon=\frac{f}{f_{0}}$,

which means that the survival factor can be used to correct the line-of-sight integrals by modifying the source distribution function according to

$f(\boldsymbol{v}, \boldsymbol{x}) \rightarrow \epsilon(\boldsymbol{v}, \boldsymbol{x}) \cdot f_{0}(\boldsymbol{v}, \boldsymbol{x})$,

where $f_{0}(\boldsymbol{v}, \boldsymbol{x})$ is the distribution function immediately on the downstream side.

Explicit spectral ENA fluxes will be calculated in a separate publication; in the remaining parts of this study, we focus on the extinction factor $\epsilon$ itself. In Fig. 6, we demonstrate "isoextinction" lines, that is, lines where a certain percentage of energetic ions has been eliminated by charge exchange. This figure clearly shows that there is only a small contribution to ENAs from flows beyond solar distances of $150 \mathrm{AU}$ in the upwind direction or beyond $350 \mathrm{AU}$ in the downwind direction, because the source population of energetic protons is exhausted at larger distances. This automatically limits the transit-time lengths that one may sample with line-of-sight integrated ENAs to 5-6 years, which is, approximately one half of a solar cycle.

\section{Applications}

\subsection{Modifications of spectral intensities}

Even without a full study of ENA fluxes, it is possible to derive some qualitative spectral results from our formalism. First, we note that the transit-time delays and all related parameters, including the ion-loss effect discussed above, depend on the energy of the ENAs observed by the detector, which in turn depends on the velocity $v_{\text {rel }}$. This effect can be discussed qualitatively, that is, without additional assumptions on the parent population $f$.

Taking the general solution of the extinction equation, Eq. (8), it follows that

$\epsilon=\frac{f}{f_{0}}=\exp \left(-n_{\mathrm{H}} \int_{0}^{t} \sigma_{\text {ex }}\left(v_{\text {rel }}(\boldsymbol{u})\right) v_{\text {rel }} \mathrm{d} t^{\prime}\right)$.

If we assume that the relative velocity $v_{\text {rel }}$ is roughly constant along the plasma flow line due to its vanishing sensitivity to $U(s)$, the extinction factor reduces to

$\epsilon=\exp \left(-n_{\mathrm{H}} \sigma_{\text {ex }}\left(v_{\text {rel }}\right) v_{\text {rel }} \cdot t\right)$,

which means that the extinction effect only depends on the travel-time of the plasma in the inner heliosheath, and the extinction factor transforms into a simple decay law,

$\epsilon(t)=\frac{f}{f_{0}}=\exp (-\lambda t)$

with the decay constant

$\lambda=n_{\mathrm{H}} \sigma_{\mathrm{ex}}\left(v_{\text {rel }}\right) v_{\text {rel }}$.

Any desired extinction time can then be calculated using standard methods, that is, a specific extinction factor $\epsilon$ is reached after a time of

$t(\epsilon)=\frac{\ln \epsilon}{\lambda}$.

The charge exchange cross-section is a function of $v_{\text {rel }}$, which can be approximated in the energy range between 1 and $10 \mathrm{keV}$ by a power law, $\sigma_{\text {ex }} \propto v_{\text {rel }}^{-k}$ (see e.g. McNutt et al. 1999), which is sufficiently close to the more recent results by Lindsay \& Stebbings (2005) for the present analyses. Therefore, the energydependent transit-time age for a certain extinction behaves as

$t\left(\epsilon, v_{\text {rel }}\right) \propto v_{\text {rel }}^{k-1}$.

The power index $k$ that appears in this relation is on the order of 0.1 , that is, extinction time shrinks with increasing energies, and more energetic ions are removed from the source by charge exchange with increasing efficiency. Considering that the solar wind plasma in the inner heliosheath is traveling with low speed independent of individual ions in the distribution function, this means that the isoextinction lines presented in Fig. 6 will be closer to the Sun for more energetic ions, resulting in shorter contributing line-of-sight lengths, and overall, a softer spectrum.

\subsection{Reflections of the solar cycle in ENA data}

Finally, we study the transit-time delays after which a certain behaviour of the solar cycle is expected to be observable in the IBEX data. We first calculated the extinction factor $\epsilon$ along a line-of-sight. From the results given in Fig. 7, it easily follows that a typical line-of-sight essentially does not sample 


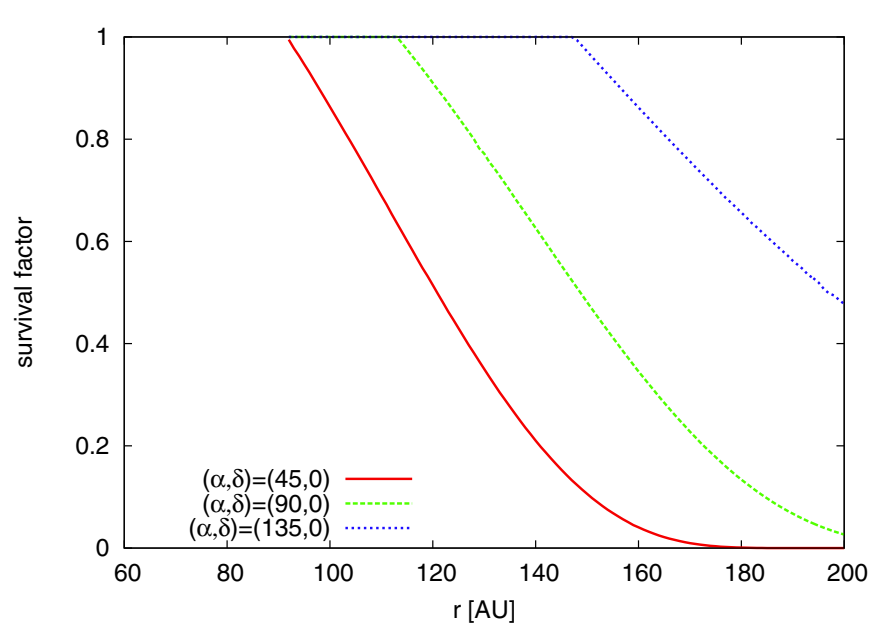

Fig. 7. Extinction coefficients along three representative line-of-sights.

ENAs over distances longer than 100 AU beyond the termination shock. However, it follows from Fig. 4 that this corresponds to a transit-time intervall of 5-6 years, with the oldest ENAs contributing the least to the total observable flux.

Without a detailed calculation, we estimate the average transit-time delay for a single point of time in the solar cycle by

$\bar{\tau}_{\mathrm{tt}} \simeq \tau_{\mathrm{sw}}+2$ years $_{-2}^{+4}$ years

where we had introduced an asymmetric uncertainty because old ENAs provide less to the line-of-sight than younger ENAs.

Finally, we need to point out that this estimate is valid for the energy band around $1 \mathrm{keV}$. At higher energies, it follows from Eq. (19) that the IBEX data will react earlier to the solar cycle, which means that the most energetic IBEX energy band probably reflects the solar cycle approximately one year earlier than the mid-energetic $1 \mathrm{keV}$ energy band. This time-scale can be resolved by IBEX, because the detector provides new skymaps every six months.

\section{Conclusions}

Several years of IBEX ENA data are now available, and with the mission continuing to produce one skymap every six months, it is becoming possible to study time-dependent effects. Understanding these effects requires a detailed understanding of how the observed ENA fluxes will react to changes in the source processes, such as the changing solar cycle or local plasma-wave interactions. Obviously, depending on different ENA source model assumptions, the reaction of the ENA fluxes to the processes that generate the energetic source proton distribution can be quite different.

We have investigated how temporal variations in the outflowing solar wind plasma may be reflected in global ENA maps that are produced by IBEX twice a year. To do this, we derived the basic formalism required to understand the reaction of ENA fluxes generated in the inner heliosheath to general dynamic processes in the Sun. In our inner heliosheath model, the transit-time that generates the total phase shift between the original timevariable solar wind structures and the resulting ENA signal is composed of three different contributions: the time $\tau_{\mathrm{p}, \mathrm{sw}}$ it takes the solar wind to advect the solar wind plasma out to the termination shock, the time $\tau_{\mathrm{p} \text {,ihs }}$ it takes the downstream solar wind to transport the shock-generated ion distribution function along the curved streamlines in the inner heliosheath until the selected ENA is generated by charge exchange with the local interstellar cold hydrogen atoms, and finally, the time $\tau_{\text {ena }}$ that is needed for this ENA to cover the return from the heliosheath source point to the IBEX detector.

We presented our results obtained in the form of energydependent isochronal lines that define contours with identical time-delays of the ENA signal from this point. Even though these results strongly depend on the solar wind speed and the flow line profile in the inner heliosheath, we have obtained similar results to earlier order-of-magnitude estimates (McComas et al. 2010, 2012b; Reisenfeld et al. 2012; Allegrini et al. 2012). Unlike these earlier results, however, we were able to consider most relevant microphysical contributions, such as the flow line profile, describing the individual contributions in each direction of the sky. To summarize, it will take at least 1-2 years for keV-energetic ENAs to start reacting to the solar cycle in the upwind direction, that is, along the stagnation line, and at least 3 years to start reacting to the solar cycle in the downwind direction. Depending on the length of the streamline along which the plasma is convected in the inner heliosheath, the delay can easily gain an additional delay of several years, which has to be considered carefully along a line-of-sight that intersects many individual streamlines in the inner heliosheath, sampling increasingly larger delay times with larger distances.

In principle, one might expect that along lines of sight directed into the tail region of the heliosphere, delay times associated with more distant ENA production regions would easily grow mmuch longer than one solar cycle period, so that in principle contributions to the detected ENA signals would average over multiple solar cycles, completely averaging out every solar cycle-induced dynamics. As we showed, however, this might not be the case, because one also has to consider ion removal effects along the plasma flow lines, which will greatly reduce the contribution of distant ENA production, because the source population of energetic ions has already been depleted by charge exchange processes. Applying this argument to a typical line-of-sight, one easily sees that the regions that generate the ENAs observed by IBEX are effectively restricted to a line-of-sight length of about $100 \mathrm{AU}$, with the exact length depending on the flow line profile in the inner heliosheath and the ENA energy. This introduces a natural cut-off to the line-of-sight integral boundaries that needs to be evaluated to calculate model predictions of ENA fluxes.

This allowed us to solve a complication with ENA modeling that was identified by, for example, Siewert et al. (2013), namely the upper limit for the line-of-sight integral in the heliotail, where the heliopause distance grows very large, reaching distances where ENA removal by reionization becomes important. In the earlier study, we had introduced a cutoff distance of $300 \mathrm{AU}$, which is quite close to the cutoff obtained here. In the tail direction, the inner heliosheath begins at solar distances of about $180 \mathrm{AU}$, and adding the $100 \mathrm{AU}$ line-of-sight length ends up surprisingly close to the ad hoc value adopted earlier.

Owing to a fundamental drawback of all fluid descriptions found in the literature, it is not possible to study this important detail exclusively in the framework of a fluid theory. Therefore, similar to this study, previous modeling attempts for instance by Heerikhuisen et al. (2007) had to assume a special distribution function for the local plasma conditions as well. To obtain reasonable fluxes of keV-ENAs, they assumed that the downstream solar wind plasma ions, instead of following a Maxwellian distribution, are distributed according to a Kappa distribution, with a constant Kappa-index all over the whole heliosheath, with a selected value of $\kappa=1.63$ that is supported by Voyager 
data. To calculate ENAs from that approach, the authors then used the steady-state solution obtained from a 3D heliospheric model based on the 3D-MHD code of Heerikhuisen et al. (2006). However, it is clear that this type of modeling, based on a constant Kappa value, results in a density-normalized local spectral intensity of energetic ions that is always the same, and thus inherently unable to describe the energy-dependent removal of energetic ions - or the boundary value for the line-of-sight integral - that we discussed here.

At $\mathrm{keV}$ energies, ions in the heliosheath undergo chargeexchange loss processes that occur more frequently than charge exchange gain processes by about the number $n_{0} / n_{j}$, where $n_{0}$ is the cold LISM neutral H-atom density $\left(o\left(0.1 \mathrm{~cm}^{-3}\right)\right)$, and $n_{j}$ is the much lower density of transcharged solar wind $\mathrm{keV} \mathrm{H}$-atoms $\left(o\left(10^{-4} \mathrm{~cm}^{-3}\right)\right)$. Therefore, the keV-energetic ion component is dominated by charge exchange losses, an effect that must be included in any realistic model of the outer heliosphere; in principle, this requirement concerns only ENA production models, because resulting ion removal will also result in a reduced ion pressure and therefore a different shape of the heliopause and the heliotail.

We are currently working on a new study by updating our earlier model calculations (Fahr et al. 2011; Siewert et al. 2012, 2013) to include these additional corrections; for this, we carefully investigate how these new effects would quantitatively modify ENA flux spectra and related properties. This also includes a modified heliosheath pressure distribution and hence as a reaction of that, the heliosheath streamline configuration.

Acknowledgements. M. Siewert is grateful to the Deutsche Forschungsgemeinschaft for financial support granted to him as part of the project Si-1550/2-2. D. J. McComas is supported by NASA's Explorers Program as a part of the IBEX mission.

\section{References}

Allegrini, F., Bzowski, M., Dayeh, M. A., et al. 2012, ApJ, 749, L41

Burlaga, L. F., Ness, N. F., Belcher, J. W., Lazarus, A. J., \& Richardson, J. D. 1996, Space Sci. Rev., 78, 33

Chalov, S. V., Fahr, H.-J., \& Malama, Y. G. 2007, Ann. Geo. Comm., 25, 575

Fahr, H.-J. 2007, Ann. Geophys., 25, 2649
Fahr, H.-J., \& Fichtner, H. 1991, Space Sci. Rev., 58, 193

Fahr, H.-J., \& Fichtner, H. 2011, A\&A, A92

Fahr, H. J., \& Ruciński, D. 1999, A\&A, 350, 1071

Fahr, H.-J., \& Siewert, M. 2013, A\&A, 552, A41

Fahr, H.-J., Siewert, M., McComas, D. J., \& Schwadron, N. A. 2011, A\&A, 531, A77

Funsten, H. O., Allegrini, F., Crew, G. B., et al. 2009, Science, 326, 964

Fuselier, S. A., Allegrini, F., Funsten, H. O., et al. 2009, Science, 326, 962

Heerikhuisen, J., Florinski, V., \& Zank, G. P. 2006, J. Geophys. Res., 111, 6110

Heerikhuisen, J., Pogorelov, N. V., Zank, G. P., \& Florinski, V. 2007, ApJ, 655, L53

Heerikhuisen, J., Pogorelov, N. V., Zank, G. P., et al. 2010, ApJ, 708, L126

Le Roux, J. A., \& Fichtner, H. 1997, ApJ, 477, L115

Lindsay, B. G., \& Stebbings, R. F. 2005, J. Geophys. Res., 110, 12213

Livadiotis, G., McComas, D. J., Dayeh, M. A., Funsten, H. O., \& Schwadron, N. A. 2011, ApJ, 734, 1

McComas, D. J., Barraclough, B. L., Funsten, H. O., et al. 2000, J. Geophys. Res., 105, 10419

McComas, D. J., Allegrini, F., Bochsler, P., et al. 2009a, Science, 326, 959

McComas, D. J., Allegrini, F., Bochsler, P., et al. 2009b, Space Sci. Rev., 146, 11

McComas, D. J., Bzowski, M., Frisch, P., et al. 2010, J. Geophys. Res. (Space Physics), 115, A09113

McComas, D. J., Alexashov, D., Bzowski, M., et al. 2012a, Science, 336, 1291

McComas, D. J., Dayeh, M. A., Allegrini, F., et al. 2012b, ApJS, 203, 1

McComas, D. J., Dayeh, M. A., Funsten, H. O., Livadiotis, G., \& Schwadron, N. A. 2013, ApJ, 771, 77

McNutt, R. L., Lyon, J., \& Goodrich, C. C. 1999, J. Geophys. Res., 104, 14803

Parker, E. N. 1965, Planet. Space Sci., 13, 9

Ratkiewicz, R., Barnes, A., Molvik, G. A., et al. 1998, A\&A, 335, 363

Reisenfeld, D. B., Allegrini, F., Bzowski, M., et al. 2012, ApJ, 747, 110

Richardson, J. D., Paularena, K. I., Lazarus, A. J., \& Belcher, J. W. 1995, Geophys. Res. Lett., 22, 325

Richardson, J. D., Kasper, J. C., Wang, C., Belcher, J. W., \& Lazarus, A. J. 2008, Nature, 454, 63

Schwadron, N. A., \& McComas, D. J. 2013, ApJ, 764, 92

Schwadron, N. A., Bzowski, M., Crew, G. B., et al. 2009, Science, 326, 966

Schwadron, N. A., Allegrini, F., Bzowski, M., et al. 2011, ApJ, 731, 56

Scudder, J. D., \& Karimabadi, H. 2013, Proc. AGU Fall Meeting 2013, abstract \#SH33D-05

Siewert, M., Fahr, H.-J., McComas, D. J., \& Schwadron, N. A. 2012, A\&A, 539, A75

Siewert, M., Fahr, H.-J., McComas, D. J., \& Schwadron, N. A. 2013, A\&A, 551, A58

Wu, P., Winske, D., Gary, S. P., Schwadron, N. A., \& Lee, M. A. 2009, J. Geophys. Res., 114, 8103

Zank, G. P., Heerikhuisen, J., Pogorelov, N. V., Burrows, R., \& McComas, D. 2010, ApJ, 708, 1092 